\title{
Quality of life four years after acute myocardial infarction: short form 36 scores compared with a normal population
}

\author{
N Brown, M Melville, D Gray, T Young, J Munro, A M Skene, J R Hampton
}

\begin{abstract}
Objectives-To assess the impact of myocardial infarction on quality of life in four year survivors compared to data from "community norms", and to determine factors associated with a poor quality of life.
\end{abstract}

Design-Cohort study based on the Nottingham heart attack register.

Setting-Two district general hospitals serving a defined urban/rural population. Subjects-All patients admitted with acute myocardial infarction during 1992 and alive at a median of four years.

Main outcome measures-Short form 36 (SF 36) domain and overall scores.

Results-Of 900 patients with an acute myocardial infarction in 1992 , there were 476 patients alive and capable of responding to a questionnaire in 1997. The response rate was $424(89.1 \%)$. Compared to age and sex adjusted normative data, patients aged under 65 years exhibited impairment in all eight domains, the largest differences being in physical functioning (mean difference 20 points), role physical (mean difference 23 points), and general health (mean difference 19 points). In patients over 65 years mean domain scores were similar to community norms. Multiple regression analysis revealed that impaired quality of life was closely associated with inability to return to work through ill health, a need for coronary revascularisation, the use of anxiolytics, hypnotics or inhalers, the need for two or more angina drugs, a frequency of chest pain one or more times per week, and a Rose dyspnoea score of $\geqslant 2$.

Conclusions-The SF 36 provides valuable additional information for the practising clinician. Compared to community norms the greatest impact on quality of life is seen in patients of working age. Impaired quality of life was reported by patients unfit for work, those with angina and dyspnoea, patients with coexistent lung disease, and those with anxiety and sleep disturbances. Improving quality of life after myocardial infarction remains a challenge for physicians.

(Heart 1999;81:352-358)

Keywords: quality of life; acute myocardial infarction; short form 36

The assessment of any disease process from a doctor's perspective is predominantly medi- cally orientated, generally focusing on specific adverse signs and symptoms thought to give a crude indication of the severity of disease or its prognosis or, following a therapeutic intervention, the success (or otherwise) in relieving symptoms. Even so, physicians may significantly underestimate patient reported symptoms of physical ill health. ${ }^{1}$

Of greater importance from the patient's perspective, however, are the limitations imposed by an episode of illness on day to day activities around the home, at leisure, and at work which adversely affect quality of life. When making a judgment on the severity of a disease process, effectiveness of treatment or overall health related outcome, it would be of value to have a more complete assessment, which would include health related quality of life. $^{2}$

Several tools are available to assess quality of life. Some have been designed to be specific to a particular disease; other "generic" questionnaires apply equally to a range of diseases and enable comparison between these and normative scores. The short form 36 (SF 36) ${ }^{3}$ and the Nottingham health profile (NHP) ${ }^{4}$ are two of the most widely evaluated. The SF 36 was developed in the US as part of the medical outcomes study and has been further tested and validated in a British population. ${ }^{5}{ }^{6}$ The NHP was developed in Nottingham from factors considered relevant by lay people, and local community norms are available, ${ }^{7}$ although this tool has been recently criticised for failing to detect lower but potentially important levels of morbidity. ${ }^{5}$

The focus of attention in the immediate period following a myocardial infarction is generally on physical functioning, but following discharge from hospital and in the longer term general health, vitality, and social and emotional functions become at least as important. Where studies are available, the impact on quality of life in the short and medium to long term after a myocardial infarction appears variable, ${ }^{8-10}$ but the populations under study are often highly selective. ${ }^{89}$

Our study aimed to determine the quality of life in an unselected cohort of survivors of a myocardial infarction using the generic SF 36 questionnaire. We wanted to assess whether our survivors' perceived health differed from "community norms" by comparing their responses with those obtained from age and sex adjusted "normative" controls; and to determine any baseline characteristics, events or interventions during follow up and current symptomatology (including Rose angina and 
Table 1 Comparison of characteristics of questionnaire responders and non-responders in four year survivors of a myocardial infarction

\begin{tabular}{|c|c|c|c|}
\hline & $\begin{array}{l}\text { Responders } \\
(n=421)\end{array}$ & $\begin{array}{l}\text { Non-responders } \\
(n=64)\end{array}$ & $p$ Value \\
\hline Median age (IQR) & $67(59,73)$ & $68(56,78)$ & 0.602 \\
\hline Male & $278(66.0)$ & $37(57.8)$ & 0.253 \\
\hline Previous myocardial infarction & $51(12.1)$ & $10(15.6)$ & 0.557 \\
\hline Q wave myocardial infarct & $336(79.8)$ & $47(73.4)$ & 0.317 \\
\hline Anterior infarction & $165(39.2)$ & $18(26.9)$ & 0.118 \\
\hline Diabetes & $45(10.7)$ & $7(10.9)$ & 0.875 \\
\hline \multicolumn{4}{|l|}{ Killip class } \\
\hline 1 & $278(66.0)$ & $49(76.6)$ & 0.126 \\
\hline 2 & $124(29.5)$ & $12(18.8)$ & 0.104 \\
\hline $3 / 4$ & $17(4.0)$ & $3(4.7)$ & NA \\
\hline \multicolumn{4}{|l|}{ Events after discharge (admissions) } \\
\hline Coronary revascularisation & $67(15.9)$ & $4(6.3)$ & 0.046 \\
\hline Recurrent myocardial infarction & $46(10.9)$ & $7(10.4)$ & 0.832 \\
\hline Possible infarction/unstable angina & $76(18.1)$ & $10(15.6)$ & 0.766 \\
\hline Cardiac failure & $25(5.9)$ & $3(4.7)$ & NA \\
\hline
\end{tabular}

Values are $\mathrm{n}(\%)$ unless stated otherwise.

$\mathrm{IQR}$, interquartile range; NA, not applicable (numbers too small for $\chi^{2}$ to be valid).

dyspnoea scores) ${ }^{11}$ or medications that may underlie any impaired health related quality of life.

\section{Methods}

PATIENT COHORT

The methods of data collection for the Nottingham heart attack register have been previously described in detail elsewhere. ${ }^{12}$ Briefly, all patients admitted to Nottingham's two hospitals with symptoms suggestive of acute myocardial infarction are identified on admission and an extensive record of management and in-hospital outcome documented. All patients satisfying the criteria for an acute myocardial infarction (a compatible history plus either diagnostic changes of ST segment elevation evolving new $\mathrm{Q}$ waves in the ECG or a rise in cardiac enzymes to greater than twice the upper limit of normal, or both) during 1992 and who survived to discharge were included in this analysis.

The last known address and general practitioner of each patient in this cohort was retrieved from the Nottingham district health authority records. Details of each patient's management and outcome over the four years following the index myocardial infarction were determined retrospectively from a combination of review of all hospital medical records and the general practitioner notes, the coroners' reports, and information from the Office for National Statistics.

All surviving patients at a median of four years were invited to complete and return by post a detailed questionnaire (described below). A covering letter in large type was enclosed; this outlined the purpose of the study, advised that a patient could have help to complete the form (but requested that where appropriate this should be documented), and included a contact number for inquiries. A reminder letter and second questionnaire was sent to non-responders after two weeks.

QUESTIONNAIRE

The questionnaire was in four parts: a list of basic demographic questions; the Rose angina and dyspnoea scales ${ }^{11}$; the SF 36 health survey; and the NHP. A comparison between the SF 36 and NHP will be reported separately.

The UK standard version of the SF 36 was administered in this survey. This consists of 36 items (questions) grouped into eight multiitem domains, measuring the following:

(1) Physical functioning (10 items)

(2) Social functioning (two items)

(3) Role limitations because of physical problems (role physical) (four items)

(4) Role limitations because of emotional problems (role emotional) (three items)

(5) Mental health (five items)

(6) Energy/vitality (four items)

(7) Bodily pain (two items)

(8) General health perception (five items).

The $36^{\text {th }}$ question inquired about change in health in the last year; for the purposes of this study, the results are available but not reported here. Each of the scores for the domains were coded, summed, and transformed on to a scale from 0 (worst possible health) to 100 (best possible health).

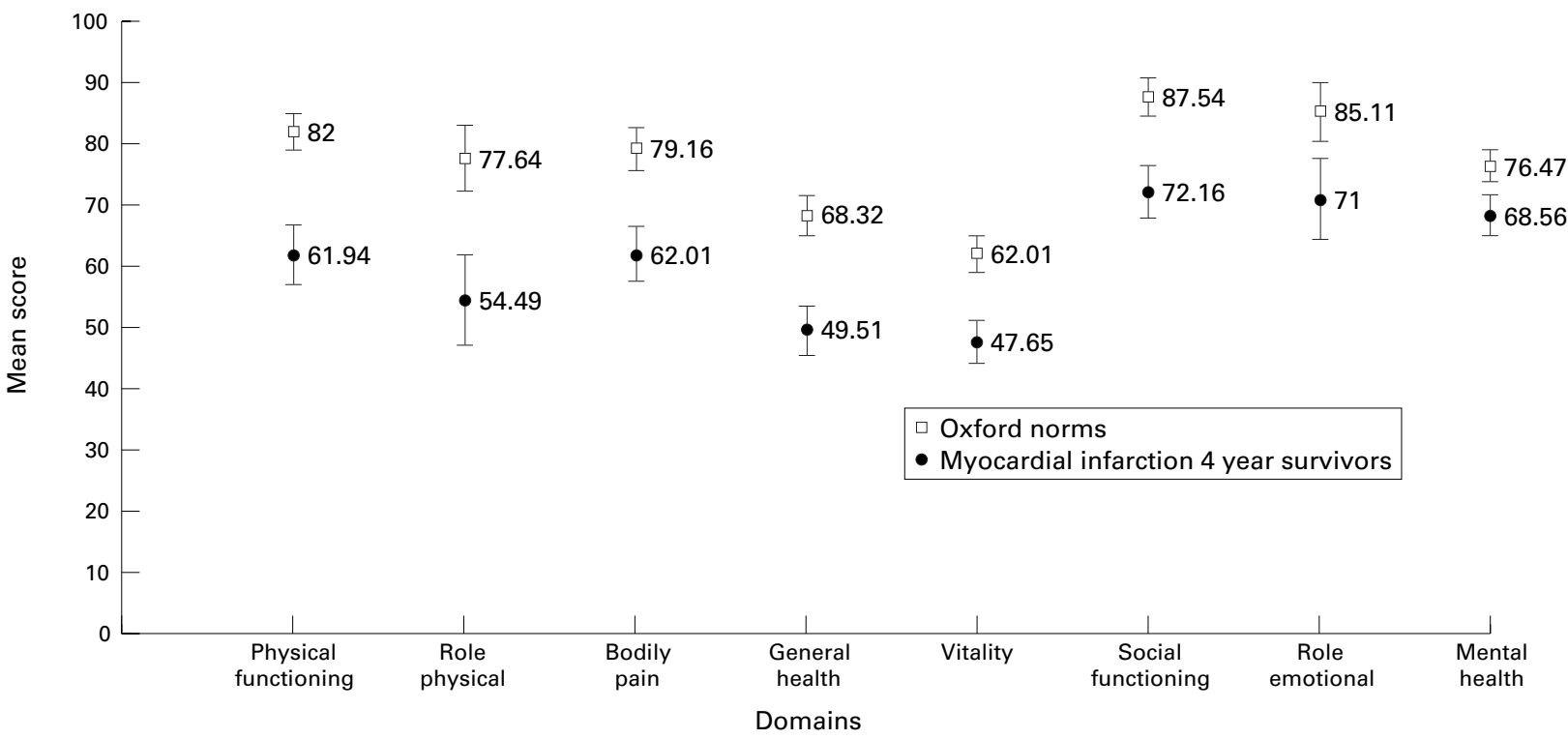

Figure 1 Mean (95\% CI) SF 36 scores for four year myocardial infarction survivors aged under 65 years compared to age and sex adjusted Oxford norms. 


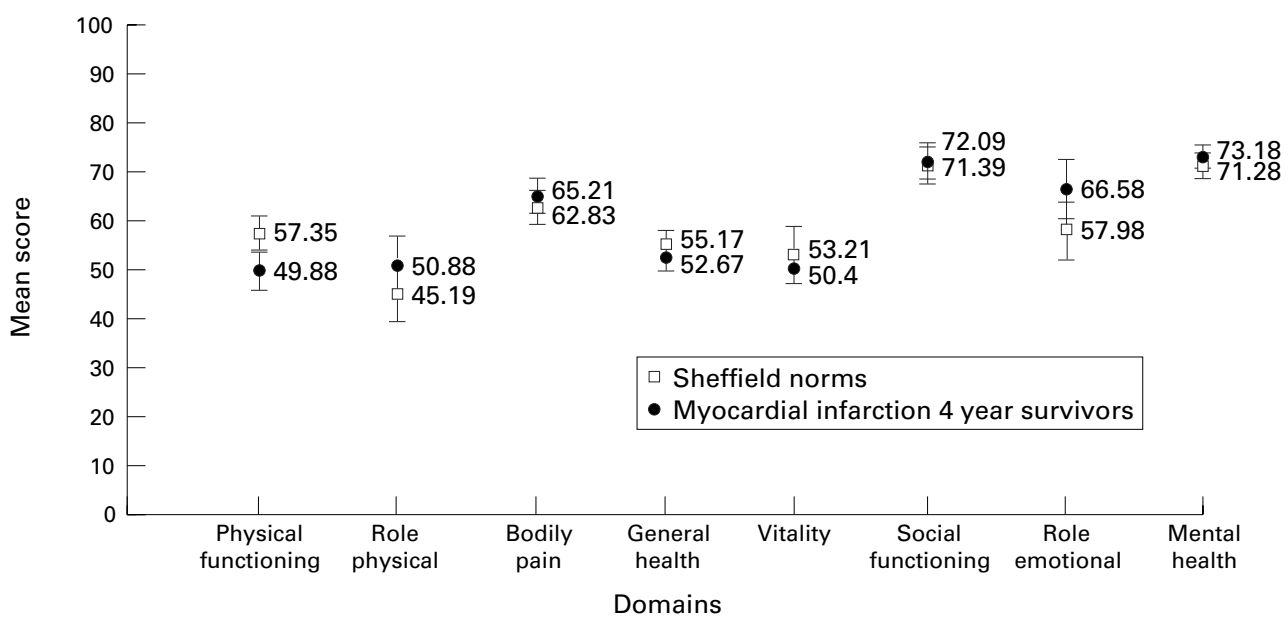

Figure 2 Mean (95\% CI) SF 36 scores for four year myocardial infarction survivors aged over 65 years compared to age and sex adjusted Sheffield norms.

COMPARISON WITH NORMATIVE POPULATIONS To provide some estimate of the potential impact of a myocardial infarction on medium to long term outcome, the results obtained from the SF 36 quality of life questionnaire were compared with data on quality of life from two normative data sets. Study respondents under 65 were compared with normative data from Oxford. ${ }^{13}$ The authors of the Oxford norms were concerned about the higher level of missing data in the elderly as reported by Brazier and colleagues ${ }^{5}$ and have provided data for adults of working age only. Modifying the layout of the questionnaire to include a larger type and allowing assistance has resulted in successful use of the SF 36 in the elderly. ${ }^{14}$ Accepting these limitations we compared those over 65 years old with baseline SF 36 scores from 8117 respondents to a postal survey of 10619 patients randomly selected from 12 general practices in Sheffield to be potentially eligible for a randomised trial of exercise in the elderly ( $\mathrm{J}$ Munro, unpublished data).

\section{STATISTICAL ANALYSIS}

The $\chi^{2}$ test and Wilcoxon sign rank test were used in a comparison of the background characteristics of responders and nonresponders.

Non-parametric tests were used to compare between group differences. Multiple linear regression models were fitted to the data for each of the eight domains of the SF 36 and an overall quality of life score in order to identify factors affecting quality of life in four year survivors of a myocardial infarction. The overall measurement of quality of life, using the results from the SF 36, was calculated using principal components analysis. ${ }^{15}$ As the domain scores were all highly and positively correlated with each other, this technique was used to simplify multivariate analysis by taking the set of eight correlated domains and reducing them to a smaller number of uncorrelated variables (principal components). The first principal component was selected for further modelling as this explained the majority of variation of the original scores for the eight SF 36 domains.
The first principal component represents a weighted average of the eight domains of the SF 36, with equal importance being given to all domains except mental health where slightly less weighting is placed. Baseline characteristics such as age, sex, type of infarction, severity, events after discharge such as reinfarction, rehospitalisation, return to work, current symptoms, and treatments were considered in the model. Cardiac rehabilitation was not included in the model, as programmes of rehabilitation have not been shown to have an impact on quality of life beyond the short term. ${ }^{16}{ }^{17}$ Furthermore a significant proportion of this unselected cohort did not receive an invitation to attend rehabilitation primarily because of management on a general medical ward.

All tests were two tailed with $\mathrm{p}<0.05$ considered significant. The S-Plus ${ }^{18}$ and GLIM $^{19}$ computer packages were used for all statistical analysis.

\section{Results}

PATIENT COHORT

During 1992, 960 patients were admitted who proved to have an acute myocardial infarction. Of these, 60 patients were excluded from further study: 21 because the index admission had been for a second episode of myocardial infarction that year; and 39 because they lived outside the Nottingham health district or had been only temporarily resident, or had been miscoded. Two hundred and five patients $(23.8 \%)$ died in hospital during the index admission. Of the 695 who survived to be discharged, follow up data were obtained on all but one patient. Over a median of four years, a further 210 patients died (mortality $16 \%$ in the under $65 \mathrm{~s}$ and $42 \%$ in the over $65 \mathrm{~s}$ ), leaving 485 survivors. Of these, nine patients were found to have serious mental handicap or psychiatric illness, which prevented them from completing the questionnaire, and they were classified as non-responders. The remaining 476 patients were sent the study questionnaire. 

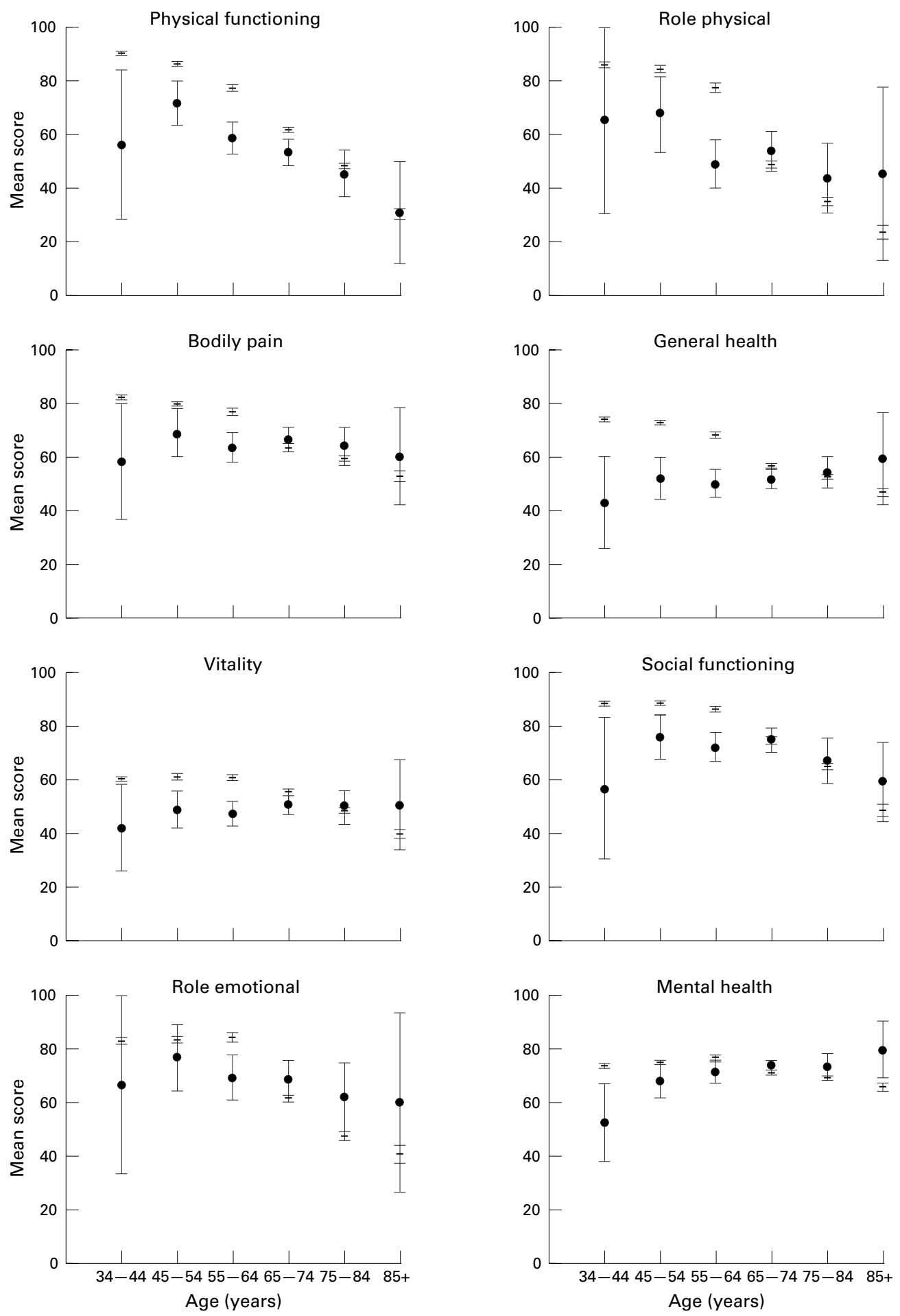

- Normative data; $\bullet 4$ year AMI survivors

Figure 3 Mean (95\% CI) age specific SF 36 domain scores for four year myocardial infarction survivors compared to normative data.

RESPONSE RATE TO QUESTIONNAIRE

A total of $424(89.1 \%)$ patients returned the questionnaire of whom 421 (88.4\%) completed the SF 36. There were no significant differences in response rates to the questionnaire in those under and those over 65 years ( $86.1 \%$ and $90.4 \%$, respectively), although the response rates to the separate domains within the SF 36 were slightly lower in those over 65 (missing domain scores varying from $1.2 \%$ to
$22 \%$ ) compared to those under 65 (missing domain scores varying from $0 \%$ to $15 \%$ ).

Fifty four patients reported that they had required some assistance to complete the questionnaire; the proportion of patients under 65 needing assistance was no different from those over 65 (19 (11.4\%) v 35 (13.8\%), p = 0.567).

Table 1 shows that there were no significant differences in the demographic characteristics of responders and non-responders. 
Table 2 Mean differences in order of importance in scores for variables associated with poor overall quality of life in a multiple regression model

\begin{tabular}{|c|c|c|}
\hline & $\begin{array}{l}\text { Mean difference in } \\
\text { overall SF } 36 \text { score }\end{array}$ & p Value ${ }^{\star}$ \\
\hline $\begin{array}{l}\text { Current unemployment because of ill health compared to } \\
\text { return to work }\end{array}$ & -32.3 & $<0.001$ \\
\hline Inhalers/no inhalers & -26.9 & $<0.001$ \\
\hline $\begin{array}{l}\text { Dyspnoea score } \\
\quad \geqslant 2 v 0 \text { or } 1\end{array}$ & -22.7 & $<0.001$ \\
\hline $\begin{array}{l}\text { Number of antianginal drugs } \\
\geqslant 2 v 0 \text { or } 1\end{array}$ & -7.6 & $<0.001$ \\
\hline $\begin{array}{l}\text { Frequency of chest pain } \\
\quad \geqslant 1 \text { episodes per week } v<1\end{array}$ & -16.4 & 0.001 \\
\hline Anxiolytics $v$ no anxiolytics & -19.3 & 0.001 \\
\hline Antiarrhythmics $v$ no antiarrhythmics & -10.4 & 0.020 \\
\hline Need for coronary revascularisation $v$ no need & -8.9 & 0.03 \\
\hline
\end{tabular}

CARDIOVASCULAR SYMPTOMS: ANGINA, DYSPNOEA SCORES

The response rates to the Rose angina and breathlessness questionnaires were 414 $(84.8 \%)$ and 407 (83.45), respectively. Four years after the acute admission 46 (11.1\%) patients had grade 1 angina, 42 (10.1\%) grade 2 angina, $71(17.1 \%)$ exertional chest pain not satisfying all four additional criteria for Rose angina (possible angina), ${ }^{20}$ and 74 (17.9\%) non-exertional chest pain. Only 181 (43.7\%) survivors reported that they were free from chest pain symptoms.

Of all 159 patients with exertional chest pain, $42(26 \%)$ had pain less than once per week, 57 (36\%) had pain one to two times per week, 51 (32\%) has pain three or more times per week, and nine patients did not record frequency. Eighty eight (55\%) of these patients were not under hospital follow up.

One hundred and fifty nine (39.1\%) patients had no breathlessness, $74(18.2 \%)$ had grade 1 dyspnoea, and 174 (42.8\%) had dyspnoea of grade 2 or more. One hundred and one (58\%) patients with a dyspnoea grade of 2 or more were not under hospital follow up.

COMPARISON OF SF 36 SCORES WITH NORMATIVE POPULATION DATA

Under 65 years age group

Figure 1 shows the mean scores (and their 95\% confidence intervals) of four year myocardial infarction survivors under 65 years, compared with normative data from Oxford, adjusted to be similar in terms of age and sex to the Nottingham myocardial infarction patients.
Significantly lower scores, implying poorer quality of life, were seen in myocardial infarction survivors in all domains, but especially those with a predominantly physical element: physical functioning (mean difference 20), role physical (mean difference 23), general health, (mean difference 19), vitality (mean difference 14), bodily pain (mean difference 17), and social functioning (mean difference 14). Smaller, but still significant, differences were seen in the domains role emotional and mental health-here the confidence intervals were closer but did not overlap.

\section{Over 65 age group}

Figure 2 shows the mean (95\% CI) scores for myocardial infarction survivors compared to normative data from Sheffield for those over 65 years, adjusted to be similar in terms of age and sex to the Nottingham myocardial infarction patients. This shows that the normative scores in patients of retirement age are similar to four year survivors of acute myocardial infarction.

The domain scores in the study population and equivalent data for the two normative populations combined, by 10 year age bands, are shown in fig 3. This shows an age related gradient with the lowest scores compared to normative data in the youngest myocardial infarction survivors.

The only patients with a significantly lower domain score in the elderly were those aged 65-74 years who had a slightly lower score in physical functioning; the study and normative populations for the elderly are otherwise indistinguishable. Differences between study and normative populations were similar for both men and women although confidence intervals were wider because of lower numbers (data not shown).

\section{FACTORS INFLUENCING QUALITY OF LIFE}

Table 2 shows the factors in multivariate analysis that were associated with impaired overall quality of life in decreasing order of importance. Mean differences in overall scores (calculated using principal components analysis) between patients with or without the characteristic or treatment found to be significant in the model are shown. The overall score was rescaled for the purposes of the table to range

Table 3 Relation between number of antianginal drugs, frequency of pain, and dyspnoea grade with mean (SD) domain scores in four year survivors of myocardial infarction - univariate analysis

\begin{tabular}{|c|c|c|c|c|c|c|c|c|}
\hline & Physical functioning & Role physical & Bodily pain & General health & Vitality & Social functioning & Emotional role & Mental health \\
\hline \multicolumn{9}{|l|}{ Number of antianginal drugs } \\
\hline $0(\mathrm{n}=151)$ & $57.8(32.5)$ & $58.6(45.0)$ & $64.3(29.2)$ & $55.2(24.4)$ & $53.0(22.6)$ & $75.2(29.0)$ & $71.1(42.0)$ & $72.5(18.6)$ \\
\hline $1(\mathrm{n}=179)$ & $56.0(32.8)$ & $49.8(44.9)$ & $64.5(29.9)$ & $51.9(23.2)$ & $49.1(23.1)$ & $72.7(30.4)$ & $66.8(42.0)$ & $72.4(20.1)$ \\
\hline $2(\mathrm{n}=55)$ & $45.3(33.7)$ & $50.0(48.7)$ & $54.6(27.5)$ & $41.8(21.7)$ & $42.0(22.8)$ & $64.4(31.0)$ & $63.9(43.5)$ & $64.8(16.6)$ \\
\hline $3(\mathrm{n}=16)$ & $42.9(22.4)$ & $32.1(45.4)$ & $54.5(26.7)$ & $42.8(26.2)$ & $41.3(25.1)$ & $64.4(31.0)$ & $63.9(43.5)$ & $64.8(16.6)$ \\
\hline $\mathrm{p}$ value & 0.055 & 0.719 & 0.091 & 0.007 & 0.022 & 0.056 & 0.941 & 0.039 \\
\hline \multicolumn{9}{|c|}{ Episodes of chest pain (per week) } \\
\hline$<1(\mathrm{n}=43)$ & $53.0(25.4)$ & $53.1(43.9)$ & $65.8(24.1)$ & $50.8(23.8)$ & $54.2(17.9)$ & $74.1(29.1)$ & $65.8(42.4)$ & $71.9(19.2)$ \\
\hline 1 or $2(\mathrm{n}=57)$ & $39.7(25.1)$ & $27.9(40.4)$ & $50.8(17.7)$ & $37.4(17.9)$ & $39.6(19.9)$ & $62.9(27.7)$ & $56.9(42.6)$ & $68.2(19.5)$ \\
\hline$\geqslant 3(n=52)$ & $28.9(24.8)$ & $17.6(33.8)$ & $40.0(20.7)$ & $32.2(17.7)$ & $29.5(22.0)$ & $52.9(29.7)$ & $58.1(43.1)$ & $62.0(22.3)$ \\
\hline $\mathrm{p}$ value & $<0.001$ & $<0.001$ & $<0.001$ & $<0.001$ & $<0.001$ & 0.002 & 0.548 & 0.096 \\
\hline \multicolumn{9}{|l|}{ Dyspnoea score } \\
\hline No dyspnoea $(\mathrm{n}=152)$ & $73.6(29.7)$ & $73.9(40.3)$ & $75.2(27.8)$ & $65.8(19.6)$ & $60.8(20.8)$ & $83.1(25.5)$ & $82.6(34.6)$ & $75.7(17.9)$ \\
\hline Grade $1(n=73)$ & $61.7(25.2)$ & $56.9(43.0)$ & $66.1(28.5)$ & $55.8(21.8)$ & $54.7(17.4)$ & $83.1(20.7)$ & $73.7(38.4)$ & $77.0(16.5)$ \\
\hline Grade $\geqslant 2(n=163)$ & $35.1(26.2)$ & $29.0(40.5)$ & $50.5(25.0)$ & $37.3(19.7)$ & $37.7(21.0)$ & $58.9(30.7)$ & $53.3(44.4)$ & $65.7(20.2)$ \\
\hline $\mathrm{p}$ value & $<0.001$ & $<0.001$ & $<0.001$ & $<0.001$ & $<0.001$ & $<0.001$ & $<0.001$ & $<0.001$ \\
\hline
\end{tabular}


from 0 to 100 to enable comparison with the eight domains.

Baseline characteristics at the time of the myocardial infarction in 1992 were not found to be significantly associated with subsequent quality of life. An inability to return to work or be fit for work was associated with large differences in overall quality of life (lower mean scores). Both chest pain on at least a weekly basis and a requirement for two or more antianginal drugs were associated with worse quality of life as was a dyspnoea score of 2 or more. Use of inhalers (a surrogate marker for reversible airways disease), anxiolytics/hypnotics, and antiarrhythmics were all associated with lower quality of life. A slightly lower score was seen in overall quality of life in patients who had had coronary revascularisation $(72(88 \%)$ of whom had coronary surgery). Treatment with $\beta$ blockers was associated with a slightly better overall quality of life.

Table 3 shows the distribution in mean (SD) scores for each domain associated with increasing number of antianginal drugs prescribed, increasing frequency of chest pain, and worse dyspnoea score. Significantly lower scores are seen with increasing number of antianginal drugs needed in the domains of general health, vitality, and mental health with borderline differences in physical functioning. Increasing frequency of chest pain was associated with significantly lower scores in all domains measuring physical health but no differences in the emotional role or mental health domains. The marked influence of dyspnoea on quality of life is highlighted with significantly lower scores in all domains ( $p<0.001)$, including those measuring mental wellbeing.

\section{Discussion}

Physicians traditionally adopt a predominantly disease orientated approach to patient management, aiming to reduce mortality and morbidity and the risk of subsequent events. This is compounded by protocol driven care plans and by busy outpatient clinics that generally preclude detailed inquiry into the broader aspects of health and particularly the impact of disease on lifestyle. To what extent a myocardial infarction has impinged on the quality of a patient's lifestyle may be determined informally at best and is likely to be restricted to a brief discussion, perhaps regarding a younger patient's prospects for a speedy return to work.

Is there justification in moving away from a purely medically orientated approach to health care towards a more routine assessment of quality of life in myocardial infarction patients?

Previous work in the late 1970s in Dundee suggested important impairment in social and leisure activities in almost $50 \%$ of a small group of 59 survivors four years after a myocardial infarction. ${ }^{10}$ Following this, using the NHP, quality of life has been reported to be relatively good five years after myocardial infarction in Sweden with a small impairment in the domains of energy, sleep, and mobility when compared to normative data. ${ }^{9}$ However, the patients in this study were originally participants in a $\beta$ blocker trial and therefore a selected population. More recently, in a cohort of thrombolytic trial participants in Australia ${ }^{8}$ quality of life has been described as "generally high" six months after infarction using the York health measurement questionnaire. Because of selection bias associated with clinical trials, these recent reports could be underestimating impairment of perceived health. Finally, Westin et al, in a prospective study of quality of life in patients under 70 years of age with previous infarction from a coronary care registry, showed residual problems with quality of life at one year. ${ }^{21}$

In our study, the Rose angina and dyspnoea questionnaires showed that at least half of those patients who survive an acute myocardial infarction continue to experience symptoms attributable to coronary artery disease four years later; these might have been identified during routine symptomatic inquiry in clinic assuming they were still under active follow up. Without specific questioning concerning fitness for work, frequency of pain, concurrent treatment including anxiolytics, comorbidity, and an assessment of quality of life, the impact of coronary artery disease on patients' perceived health would not have been appreciated. Patients with symptoms of angina only once or twice a week, which clinicians might not consider to have much impact on lifestyle, had significantly lower quality of life scores as did those taking two or more drugs for angina. Other workers have previously shown the impact of angina on perceived health to be important, even in those regarded as stable and not being considered for further intervention. ${ }^{22}$ Furthermore, in our study, according to data from the SF 36, those aged under 65 years experience a quality of life which is considerably worse than that of similarly aged people who have not had an infarct, while those aged over 65 years seem little different from their peers.

The differences in several SF 36 domain scores for the under $65 \mathrm{~s}$ were not small. The designers of the questionnaire have suggested a benchmark of around 20 or more points as being clinically important, since in their original work differences of this order in the physical components of quality of life distinguished between patients with minor and serious medical conditions. ${ }^{23}$

Why do infarct survivors over 65 report similar quality of life to those who have not sustained a myocardial infarction? Comorbidity, particularly arthritis and chronic lung disease, are more common with advancing age. As the normative data show, there is a decline in most domains with increasing age, particularly but not exclusively those with a physical component. Both in-hospital and longer term mortality after myocardial infarction is high in the older age groups. It is therefore conceivable that four year survivors may be little different from their peers, indicating a "healthy survivor effect". In addition it has been shown in other disease states that the expectations of the elderly and the degree of dissatisfaction at a given level of disability is less than in younger individuals. ${ }^{24}$ Nevertheless patients between 
the ages of 65 and 74 years do experience some impairment with slightly lower scores in the physical functioning domain.

Previous criticism has highlighted regional differences in SF 36 scores; however, comparable Oxford and Sheffield data for the same age groups are notable for similarities in domain scores. ${ }^{25}$

Quality of life at four years is associated with the ability to return to work or be fit for and actively seeking work. It is likely that poor health determines both quality of life and the ability to work. Patients requiring coronary revascularisation had a slightly worse quality of life but they are likely to have been the most somatically affected preoperatively and we have no baseline quality of life data on this group before intervention. Others have shown persistence of distress one year after successful coronary bypass grafting. ${ }^{21}$ In agreement with others, ${ }^{9}{ }^{26}$ symptoms of both angina and dyspnoea were very important determinants of quality of life. Multiple regression analysis showed frequency of pain and number of antianginal drugs to be more predictive of poor quality of life than the presence of Rose angina. This highlights the potential deficiencies of this tool, which may only recognise those with classic symptoms. ${ }^{27}$ As both use of inhalers (surrogate marker for airways disease) and Rose dyspnoea grade were significant independent variables in the overall quality of life model, it is likely that dyspnoea of cardiac and respiratory origin are relevant in this cohort.

Our findings suggest that in an unselected cohort of medium term survivors of a myocardial infarction, a notable adverse effect on quality of life, particularly in younger patients, could go unrecognised. In view of the high response rate of $88 \%$ the SF 36 seems both acceptable to cardiac patients and a useful and sensitive tool for quality of life assessment, providing valuable information not identified in routine clinical evaluation.

Whether a more aggressive pattern of follow up and treatment, as suggested by others, ${ }^{28}$ might improve quality of life remains to be seen and warrants a well designed prospective study.

We are grateful to John Brazier from Sheffield for comments on the study and paper. We are also grateful to the staff of the register for continued support and the general practitioners of Nottingham for their cooperation with this project. The Nottingham heart attack register has been supported by the UK Department of Health since its inception in 1973.

1 Calkins DR, Rubenstein LV, Cleary PD, et al. Failure of physicians to recognize functional disability in ambulatory patients. Ann Intern Med 1991;114:451-4.
2 Wenger NK, Mattson ME, Furberg CD, et al. Assessment of quality of life in clinical trials of cardiovascular therapies. New York: Le Jacq, 1984

3 Ware JE Jr, Sherbourne CD. The MOS 36-item short-form health survey (SF-36). I. Conceptual framework and item selection. Med Care 1992;30:473-83.

4 Hunt SM, McEwen J, McKenna SP. Measuring health status. London: Croom Helm, 1986.

5 Brazier JE, Harper R, Jones NMB, et al. Validating the SF-36 health survey questionnaire: new outcome measure for primary care. $B M \mathcal{F} 1992 ; 305: 60-164$

6 Ruta D, Garratt A, Abdalla M, et al. The SF 36 health survey questionnaire: a valid measure of health status. BMF 1993;307:448-9.

7 Hunt SM, McEwen J, McKenna SP. Perceived health: age and sex comparisons in a community. 7 Epidemiol Community Health 1984;38:156-60.

8 Glasziou PP, Bromwich S, Simes RJ. Quality of life six months after myocardial infarction treated with thrombolytic therapy. AUS-TASK group. Australian arm of international tPA/SK mortality trial. Med f Aust 1994;161: 532-6.

9 Wiklund I, Herlitz J, Hjalmarson A. Quality of life five years after myocardial infarction. Eur Heart f 1989;10:464-72.

10 Finlayson A, McEwen J. Coronary heart disease and patterns of living. London: Croom Helm, 1977.

11 Rose GA. Cardiovascular survey methods, 2nd ed. Geneva: World Health Organisation, 1982.

12 Gray D, Keating NA, Murdock J, et al. Impact of hospital thrombolysis policy on out-of-hospital response to suspected myocardial infarction. Lancet 1993;341:654-7.

13 Jenkinson C, Coulter A, Wright L. Short form 36 (SF 36) health survey questionnaire: normative data for adults of working age. BMF 1993;306:1437-40

14 Singleton N, Turner A. Measuring patients' views of their health: SF 36 is suitable for elderly patients. $B M \mathcal{F}$ 1993;307:126-7.

15 Chatfield C, Collins AJ. Introduction to multivariate analysis. London: Chapman and Hall, 1980.

16 Jones DA, West RR. Psychological rehabilitation after myocardial infarction: multicentre randomised controlled trial. $B M F$ 1996;313:1517-21.

17 Oldridge N, Guyatt G, Jones N, et al. Effects on quality of life with comprehensive rehabilitation after acute myocardial infarction. Am f Cardiol 1991;67:1084-9.

18 S-plus guide to statistical and mathematical analysis, version 3.2. Seattle: Statistical Sciences, Inc, 1993.

19 Aitkin M, Anderson D, Francis B, et al. Statistical modelling in GLIM. Oxford: Oxford University Press, 1988

20 Cook DG, Shaper AG, Macfarlane PW. Using the WHO (Rose) angina questionnaire in cardiovascular epidemiology. Int F Epidemiol 1989;18:607-13.

21 Westin L, Carlson R, Israelsson B, et al. Quality of life in patients with ischaemic heart disease: a prospective controlled study. F Intern Med 1997;242:239-47.

22 Murdoch DL, Lawrence A, Henderson E, et al. Quality of life in chronic stable angina. Eur Heart f 1996;17:240.

23 McHorney CA, Ware JE Jr, Raczek AE. The MOS 36-item short form health survey (SF-36):11. Psychometric and clinical tests of validity in measuring physical and mental health constructs. Med Care 1993;31:247-63.

24 Ross CK, Sinacore JM, Stiers W, et al. The role of expectations and preferences in health care satisfaction of patients with arthritis. Arthritis Care and Research 1990;3:92-8.

25 Jenkinson C, Wright L, Coulter A. The SF 36 health survey questionnaire: if used within its limits (22). BMF 1993;307: 449.

26 Pocock SJ, Henderson RA, Seed P, et al. Quality of life, employment status, and anginal symptoms after coronary angioplasty or bypass surgery: 3-year follow-up in the randomized intervention treatment of angina (RITA) trial. Circulation 1990;94:135-42.

27 Berman DM, Brook RH, Lohr KN, et al. Conceptualisation and measurement of physiologic health for adults: volume 4, angina. Santa Monica, California: Rand Corp, 1981.

28 Mark DB, Naylor CD, Hlatky MA, et al. Use of medical resources and quality of life after acute myocardial infarction in Canada and the United States. $N$ Engl 7 Med 1994;331:1130-5. 